\title{
Remote-Controlled Monitoring of Flying Pests with an Electrostatic Insect Capturing Apparatus Carried by an Unmanned Aerial Vehicle
}

\author{
Koji Kakutani ${ }^{1}$, Yoshinori Matsuda ${ }^{2, *} \mathbb{D}$, Teruo Nonomura ${ }^{2}$, Yoshihiro Takikawa ${ }^{3}$, Kazumi Osamura ${ }^{4}$ \\ and Hideyoshi Toyoda ${ }^{5}$
}

check for

updates

Citation: Kakutani, K.; Matsuda, Y.; Nonomura, T.; Takikawa, Y.; Osamura, K.; Toyoda, H. RemoteControlled Monitoring of Flying Pests with an Electrostatic Insect Capturing Apparatus Carried by an Unmanned Aerial Vehicle. Agriculture 2021, 11, 176. https://doi.org/10.3390/ agriculture11020176

Academic Editor: Michele Rinaldi

Received: 23 January 2021

Accepted: 15 February 2021

Published: 20 February 2021

Publisher's Note: MDPI stays neutral with regard to jurisdictional claims in published maps and institutional affiliations.

Copyright: (c) 2021 by the authors. Licensee MDPI, Basel, Switzerland. This article is an open access article distributed under the terms and conditions of the Creative Commons Attribution (CC BY) license (https:/ / creativecommons.org/licenses/by/ $4.0 /)$.
1 Pharmaceutical Research and Technology Institute and Anti-Aging Centers, Kindai University, Osaka 577-8502, Japan; kakutani@kindai.ac.jp

2 Department of Agricultural Science, Faculty of Agriculture, Kindai University, Nara 631-8505, Japan; nonomura@nara.kindai.ac.jp

3 Plant Center, Institute of Advanced Technology, Kindai University, Wakayama 642-0017, Japan; takikawa@waka.kindai.ac.jp

4 Technical Development Unit, Panasonic Environmental Systems and Engineering Co., Ltd., Osaka 564-0062, Japan; osamura.kazumi@jp.panasonic.com

5 Research Association of Electric Field Screen Supporters, Nara 631-8505, Japan; toyoda@nara.kindai.ac.jp

* Correspondence: ymatsuda@nara.kindai.ac.jp

\begin{abstract}
The purpose of the study was to construct an electrostatic insect-capturing apparatus that could be applied to a drone (quadcopter). For this purpose, a double-charged dipolar electric field screen (DD-screen) was constructed using oppositely charged insulator tubes that was then attached to a drone. For charging, the inner surface of the tubes was coated with a conductive paste and then linked to a negative or positive voltage generator. The opposite charges of the tubes formed an electric field between them and created an attractive force to capture insects that entered the field. The DD-screen constructed here was sufficiently light to enable its attachment to a drone. The screen was hung from the drone perpendicular to the direction of drone movement, so as to receive the longitudinal airflow produced by the movement of the drone. It was positioned $1.8 \mathrm{~m}$ below the drone body to avoid the influence of the downward slipstream generated by the rotating propellers. Eventually, the drone was able to conduct a stable flight, with sufficient endurance, and captured airborne insects carried by an airflow of $8 \mathrm{~m} / \mathrm{s}$ during the flight. This study, therefore, provides an experimental basis for establishing a new method for conducting trap-based monitoring of airborne insects during remote-controlled flight through operation of a DD-screen attached to a drone.
\end{abstract}

Keywords: electric field; attractive force; double-charged dipolar electric field screen (DD-screen); quadcopter; unmanned aerial vehicle (UAV); slipstream; housefly; vinegar fly; tomato leaf miner fly

\section{Introduction}

An electric field screen is an air-shielding apparatus that harnesses the nature of an electric field [1]. Such screens have been used in various facilities to capture airborne spores of pathogens [2,3], flying insect pests [4,5], pollen grains that cause pollenosis [6], and fine smoke particles [7]. Electric field screens have been applied to various facilities, including domestic housing, hospital and school buildings, greenhouses used for crop production, warehouses and processing plants used for post-harvest crops, and animal husbandry facilities [8].

There is a wide variety of electric field screen structures that can be customised to prevent the entry of biotic and abiotic environmental nuisances. All screens have a simple, common structure, which typically includes an electric driver (an electric power source and direct current (DC) voltage generator) and a screen body. The electric driver is common to all types of electric field screen; however, the screen body may vary depending on the 
application. The screen can be classified as single-charged monopolar, single-charged dipolar, or double-charged dipolar [8]. The terms single-charged and double-charged refer to the mode of voltage application, i.e., using a single negative or positive voltage generator or using two generators, respectively.

Single-charged dipolar electric field screens (SD-screens) are used to control greenhouse pests. They consist of a layer of insulated conductor wires (ICWs: copper or iron wires) arrayed in parallel at definite intervals and linked to a voltage generator for negative charging and a grounded metal net [5]. These screens can be installed in the windows of a greenhouse [9]. The SD-screens are effective in repelling insects that reach the metal net of the screen, because the insects will avoid entering the electric field formed between the negatively charged layer of the ICWs and the grounded metal net [10]. This avoidance behaviour has been observed in 82 insect species ( 45 genera, 42 families, and 17 orders) [11]. A double-charged dipolar electric field screen (DD-screen), which consists of a layer of parallel ICWs that are oppositely charged and arranged alternately, has been used to capture insects invading a greenhouse [12,13]. The DD-screen forms an electric field between oppositely charged ICWs to create an attractive force to capture the insects that enter the electric field [14]. The attractive force generated by the DD-screen is sufficiently strong that it prevents the captured insects from escaping the trap.

A drone is an unmanned aerial vehicle (UAV) that is controlled remotely by a human operator or autonomously by an onboard computer. The use of UAVs has increased in response to specific demands, such as large-scale mapping, real-time assessments, and monitoring activities in ecological and environmental research fields. This has been achieved through the use of thermal, multispectral, and hyperspectral sensors combined with high-resolution geo-tagged aerial photographs and high-definition videos [15-18]. In agricultural research fields, drone-mediated aerial photography has enabled precision agricultural design $[19,20]$, phenological observation of a deciduous forest and crop yield forecasts based on tree canopy geometry [21], hyperspectral analysis of crop water stress estimation [22], and plant pest surveillance and crop yield estimation in vineyards [23]. Crop health monitoring has also been conducted by UAV-assisted dynamic clustering of wireless sensors and networks [24]. In addition to these aerophotographic approaches, UAVs have also been used to spray insecticides for pest control $[25,26]$.

UAV technology can provide farmers with a bird's-eye view of their operation and allow them to make crucial management decisions in real time. The recent progress made in the use of this technology encouraged agricultural organizations in Nara Prefecture, Japan, to plan drone-based surveillance of spotted wing drosophila (Drosophila suzukii Matsumura) in blueberry orchards. This species attacks fresh fruit and has become a severe pest of soft fruits, including strawberry, cherry, blackberry, blueberry, raspberry, and grape [27]. Direct trapping of airborne insects has been conducted to predict pest movements to neighbouring orchards. Drones can be equipped with optical instruments for aerial photography to conduct these projects [28]. Unfortunately, a drone-attachable apparatus for trapping airborne insects was not sufficiently well developed, although position-fixed traps for this pest were established [29]. Following a request from the agricultural organisations, we attempted to improve the DD-screen for this purpose. Application of the DD-screen, with its prominent insect-capturing ability, could be a feasible approach to construct a drone-carried electrostatic instrument to collect airborne insects.

The first challenge in this study was to lighten the DD-screen to below the permissible payload of the drone. A conventional DD-screen consists of multiple ICWs, a polypropylene frame, two voltage generators, and batteries [12]. The weight-changeable component was considered to be the ICW, which is constructed by passing copper or iron wires through a soft polyvinyl chloride (PVC) tube. The first way to lighten the DD-screen was to coat the inner surface of a PVC tube with a conductive paint, instead of inserting a metal wire into a tube. The next action taken was to maximise the potential of the DD-screen to capture airborne insects during the drone flight, which was achieved using flies with different body sizes as model insects. The attractive force of a DD-screen is generated in a static electric 
field (the space between the oppositely charged ICWs), and its strength is determined by the negative and positive voltages applied to the ICWs (i.e., potential difference between the ICWs) and the distance between them (pole distance of opposite charges) [14]. These factors were optimised to generate an attractive force strong enough to counter the airflow caused by the drone flight. The final aim of the study was to determine a way to attach the DD-screen to the drone. The simplest mode of attachment was to hang the DD-screen from the body of the drone. We determined the ideal positioning of the DD-screen from the drone, which exerted no influence on balance in flight and had no effect on insect capture due to the convective air current produced by the propellers of the drone. Based on the results obtained, we developed a remote-controlled electrostatic instrument to capture airborne insects.

\section{Materials and Methods}

\subsection{A Quadrotor Drone (Quadcopter)}

The drone used in this study was a quadcopter (Mavic Pro Platinum; distance between propeller shafts, $33.5 \mathrm{~cm}$; arm length, $12 \mathrm{~cm}$; weight, $734 \mathrm{~g}$; DJI, Tokyo, Japan) (Figure 1). The drone was used as an aerial vehicle to carry the DD-screens. This type of drone is capable of a controllable flight distance (cruising radius) of $4 \mathrm{~km}$, a maximum flight speed of $64.8 \mathrm{~km} / \mathrm{h}$, flight endurance (the duration time of a flight) of $27 \mathrm{~min}$ under no-load conditions, and a maximum payload of $1.1 \mathrm{~kg}$.

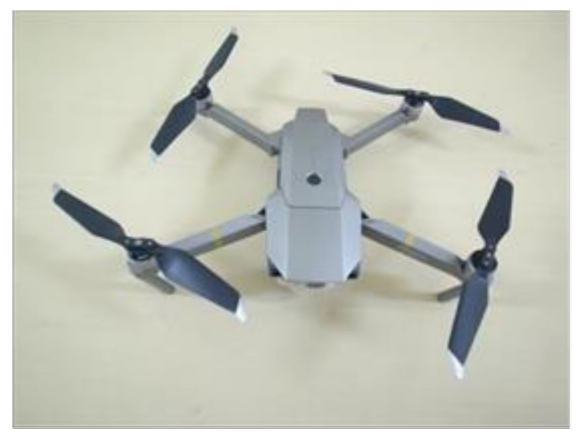

Figure 1. The quadrotor drone (Mavic Pro Platinum) used in the study.

\subsection{Measurement of Airflow Speed}

We focused on two types of airflow produced by the quadcopter: the downward slipstream (downwash) produced by the rotation of the propellers (Figure 2A) [30] and the horizontal and longitudinal airflow (L-airflow) produced by the movement of the drone (Figure 2B), which occurred in the opposite direction to that of the drone movement and passed through the DD-screen that was hung below the drone. Airborne insects in the L-airflow were captured by the DD-screen during flight. The airflow speed was measured with a vane anemometer (SK-93F II; Sato Keiryoki MFG, Tokyo, Japan). The anemometer was held at the end of a stainless-steel pole that could be extended between 1 and $3 \mathrm{~m}$, allowing it to be placed in the desired measurement position. In the first experiment, the drone hovered at a height of $3 \mathrm{~m}$, which was the lowest height with no rebound of the slipstream from the ground, and then the anemometer was positioned at the locations specified in Figure 2A to examine changes in the speed of the slipstream when the position of the anemometer was changed (between 0.5-2 $\mathrm{m}$ from the drone body). In the second experiment, the L-airflow was measured. In this experiment, the L-airflow was produced by a blower (blade length, $15 \mathrm{~cm}$ ) (Honobe Electricity, Osaka, Japan) (Figure 2B). While the drone hovered at a height of $3 \mathrm{~m}$, the horizontal airflow was recorded by an anemometer positioned at locations at different distances (0.5-2 $\mathrm{m}$ ) from the central portion of the drone body. The position of the blower was adjusted relative to the position of the anemometer to maintain a horizontal airflow. The rotation rate of the blower was adjusted with a voltage transformer (Yamabishi Denki, Tokushima, Japan) to 
generate L-airflows of 2, 5, and $8 \mathrm{~m} / \mathrm{s}$ at the position of the anemometer in the absence of slipstream. The experiment was conducted under windless conditions in a gymnasium. The volumetric flow rate $\left(\mathrm{m}^{3} / \mathrm{min}\right)$ of the L-airflow was calculated using the equation $\mathrm{Q}\left(\mathrm{m}^{3} / \mathrm{min}\right)=\mathrm{V}\left(\mathrm{m}^{2}\right) \times \mathrm{A}(\mathrm{m} / \mathrm{s}) \times 60(\mathrm{~s})$. The experiment was repeated five times, and the results were recorded as the mean and standard deviation (SD). The significance of the data was analysed statistically, as described in the caption to the table.

A
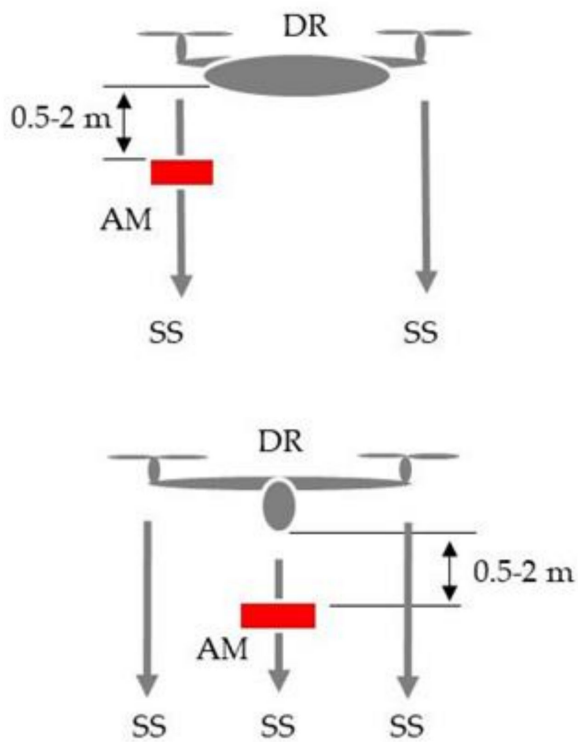

B
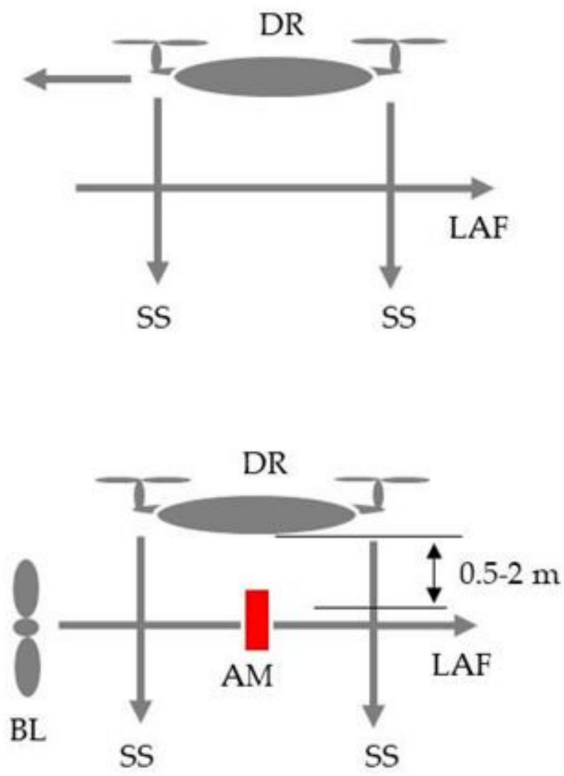

Figure 2. (A) Side (upper) and front view (lower) of a drone carrying an anemometer positioned at the front of the drone to receive the downward-directed airflow. (B) Side view of a forwardmoving (upper) and hovering (lower) drone carrying an anemometer positioned beneath its centre to receive horizontal airflow. Abbreviations: DR, drone; AM, anemometer; SS, slipstream produced by propellers; $\mathrm{BL}$, blower; LAF, longitudinal airflow by a blower.

\subsection{Charging of the Insulator Tube and Formation of an Electric Field}

To coat the inner surface of the insulator tube, an electro-conductive acrylic paste containing silver/copper filler (DOTITE FE-107, $10^{-4} \Omega \mathrm{cm}$; Fujikura, Tochigi, Japan) was solubilised with a mixture of toluene and ethylbenzene, injected into a PVC tube (length, $1 \mathrm{~m}$; thickness, $2 \mathrm{~mm}$; inner diameter, $2 \mathrm{~mm}$; resistivity, $10^{9} \Omega \mathrm{cm}$ ) (Junkosha Inc. Tokyo, Japan), and slowly circulated by a rotary pump for $1 \mathrm{~h}$, with non-adhered paste excreted from the tube. The adhesive was air-dried for $12 \mathrm{~h}$. Through this procedure, a conductive coating was formed on the inner surface of the tube (Figure 3A). The tube was cut into $25 \mathrm{~cm}$ fragments to fabricate the square DD-screen, with each side having a length of $30 \mathrm{~cm}$. The inner coating of the tube was charged by linking it to a negative or positive voltage generator (Max Electronics, Tokyo, Japan). In this electrostatic configuration, the inner coating linked to a positive voltage generator was deprived of free electrons that could be positively electrified, but free electrons were supplied by the tube coating, which was linked to a negative voltage generator to negatively charge it. The negative charge of the inner coating was polarised positively on the inner surface and negatively on the outer surface of the tube by dielectric polarisation, and vice versa for the positive charge [31]. An electric field formed between the opposite charges of the tubes. For comparison, Figure 3B shows an insulator tube that was dielectrically polarised by a conventional method, i.e., by inserting an iron conductor wire (diameter, $2 \mathrm{~mm}$ ) into the tube and connecting the wire to a negative or positive voltage generator [14]. 


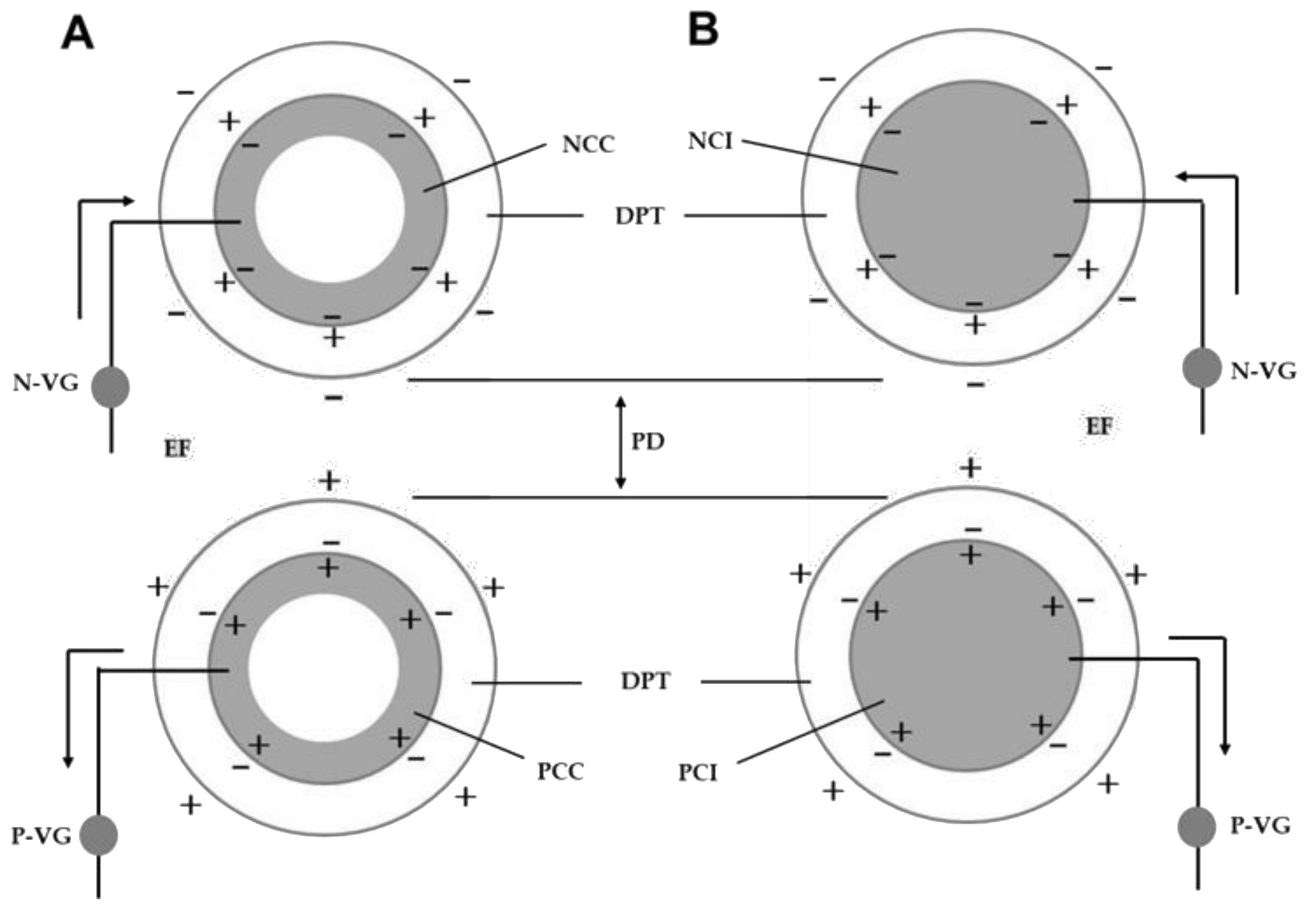

Figure 3. Schematic representation of the electric field formed by opposite charges at the surface of dielectrically polarised insulator tubes. (A): The inner surface of the tube was coated and negatively (upper) or positively (lower) charged. (B): An iron wire was inserted into a tube and negatively (upper) or positively (lower) charged. As a result of dielectric polarisation, the negative and positive charge of the inner surface coating produced negative and positive charges on the outer surfaces of the tubes, respectively. The opposite charges on the two tubes formed an electric field between them. The solid arrow indicates the direction of electricity (free electron) movement. Abbreviations: NCC, negatively charged coating of the inner surface of the tube; NCI, negatively charge iron wire; DPT, dielectrically polarised insulator tube; N-VG, negative voltage generator; PD, pole distance; EF, electric field; PCC, positively charged coating of the inner surface of the tube; PCI, positively charged iron wire; $\mathrm{P}-\mathrm{VG}$, positive voltage generator.

\subsection{Construction of the DD-Screen and Its Attachment to a Drone}

Figure $4 \mathrm{~A}$ shows the DD-screen constructed in this study. Inner-surface-coated tubes were used as DD-screen components. The tubes were arrayed in parallel at a definite interval of $5 \mathrm{~mm}$ (pole distance), alternately linked to a line of positive or negative voltage generators and fixed into a polypropylene frame. These two voltage generators were unified and placed in a box for compactness and weight reduction and then attached to the frame. The voltage generators were made to order (Max Electronics, Tokyo, Japan) and operated with three size AA rechargeable batteries connected in series (4.5 V) (duration of continuous working, $9 \mathrm{~h}$ ). We fabricated a conventional DD-screen with the same size and configuration to compare the weight of the screen. The newly constructed DD-screen was then hung from the drone.

In the experiment, the newly constructed single DD-screen was hung from the drone at different positions $(0.5-2 \mathrm{~m})$ below the centre of the drone body, as shown in Figure 4B. The screen was positioned perpendicular to the direction of movement. We examined the influence of flight speed on the flight endurance of the drone. The drone carrying the DD-screen was flown at different flight speeds $(5-20 \mathrm{~km} / \mathrm{h})$ to determine the longest flight duration at a given flight speed. To measure the endurance time ( $\mathrm{min}$ ), the drone was flown continuously until its integrated battery was drained. All experiments were conducted in a gymnasium under windless conditions. The experiment was repeated five times, and the results were recorded as the mean and standard deviation (SD). The significance of the data was analysed statistically, as described in the caption to Figure S3. 
A

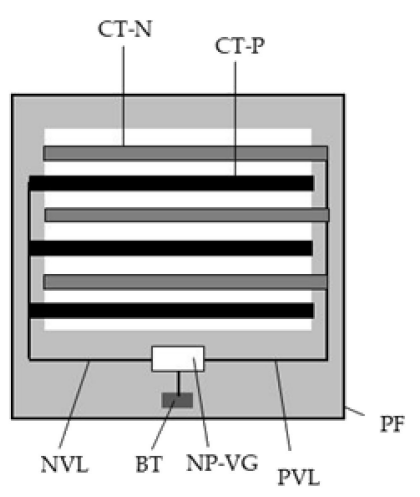

B

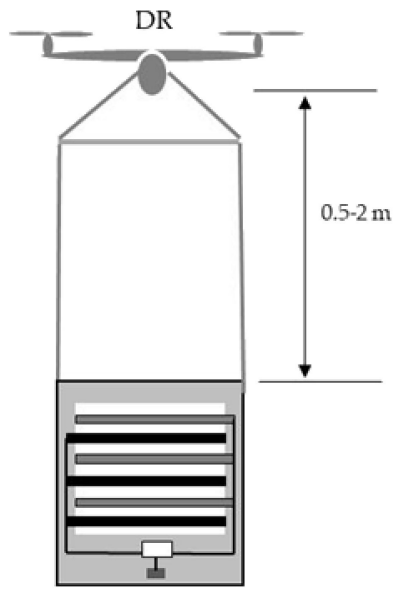

Figure 4. Configuration of a DD-screen with a voltage generator and battery $(\mathbf{A})$ and the attachment of a DD-screen to a drone (front view) (B). Abbreviations: CT-N, conductively coated insulator tube, negatively charged; CT-P, conductively coated insulator tube, positively charged; NP-VG, negative/positive voltage generators unified in a box; NVL, negative voltage line; PVL, positive voltage line; BT, battery; PF, polypropylene frame (insulator); DR, drone.

\subsection{Test Flies and Insect Capturing Assay}

Adult tomato leaf miner (Liriomyza sativae Blanchard), vinegar fly (Drosophila melanogaster Meigen), and housefly (Musca domestica Linnaeus) specimens were used in the study. Vinegar fly adults were purchased from Sumika Technoservice (Hyogo, Japan) and reared on a blue medium rusted with dried yeast (Wako Pure Chemical, Osaka, Japan) in a growth chamber $\left(25.0 \pm 0.5^{\circ} \mathrm{C}, 12 \mathrm{~h}\right.$ photoperiod at $\left.4000 \mathrm{lux}\right)$. Pupae of tomato leaf miner and housefly were also purchased and maintained in the growth chamber until adults emerged. Newly emerged adults of all test insects were collected with an insect aspirator and used in the following experiment. Houseflies, vinegar flies, and tomato leaf miners were used as models for large, medium, and small flies (L-, M-, and S-flies), respectively (Figure 5). The body sizes of these flies (length from head to wing edge) were measured using 30 randomly collected adult test insects: $3.8 \pm 0.4 \mathrm{~mm}$ (housefly), $2.9 \pm 0.3 \mathrm{~mm}$ (vinegar fly), and $1.8 \pm 0.2 \mathrm{~mm}$ (tomato leaf miner).
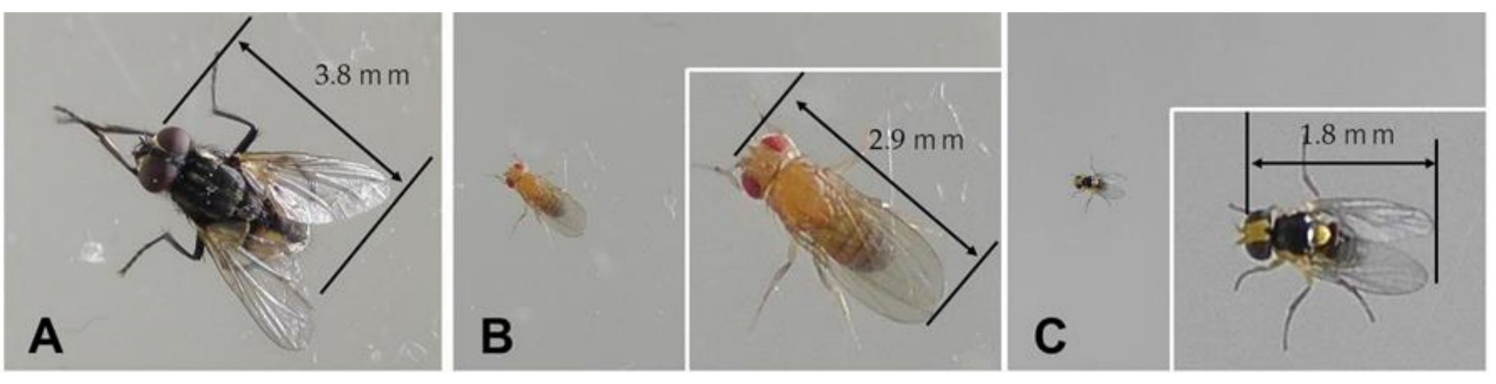

Figure 5. Comparison of the test flies with different body sizes: housefly (A), vinegar fly (B), and tomato leaf miner (C). The photographic inserts in $(\mathbf{B}, \mathbf{C})$ are enlarged images of the flies.

An insect capturing assay was conducted using the method shown in Figure 6A. In this experiment, the tubes of the DD-screen were oppositely charged with equal negative and positive voltages (between 2 and $7 \mathrm{kV}$ ), and test adult flies were collected with an insect aspirator and blown into the air stream $(2-8 \mathrm{~m} / \mathrm{s}) 40 \mathrm{~cm}$ from the screen. Insects were captured with the closest tube of the DD-screen (Figure 6B). The capture mechanism for insects in the electric field was described previously [14]. An insect was considered to have been captured completely if it remained held against the tube as it was blown on at $10 \mathrm{~m} / \mathrm{s}$ 
for $10 \mathrm{~s}$. Twenty adults were used for each voltage tested, each wind speed, and each insect species. The experiments were repeated five times, and the results were recorded as the mean and standard deviation (SD). The significance of the data was analysed statistically, as described in the caption to the table.

A

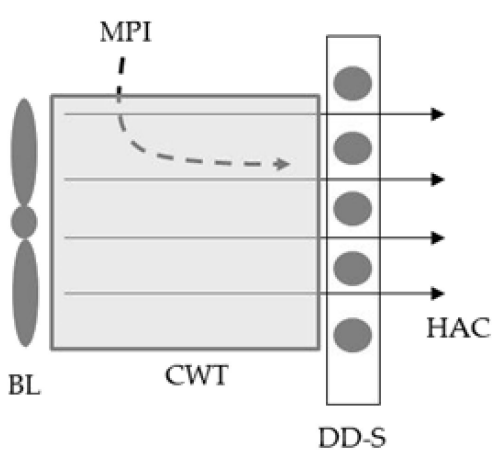

B

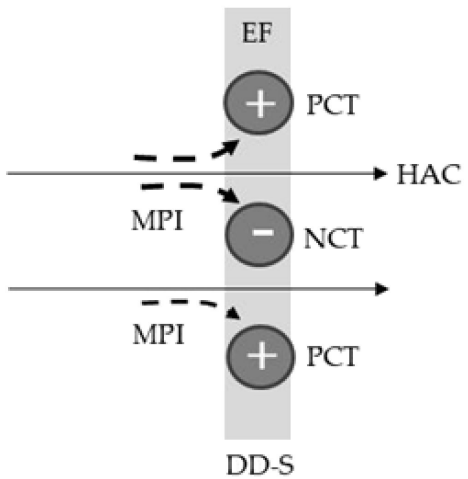

\section{C}

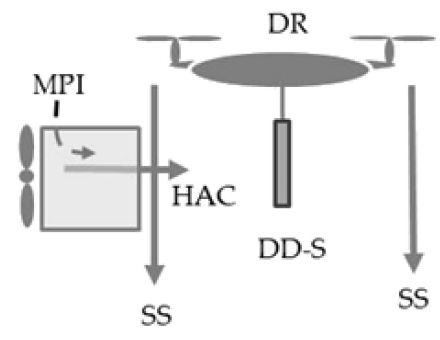

Figure 6. Side view of a DD-screen blown by a blower (A), capture of insects with negatively or positively charged tubes of the DD-screen (B), and application of the insect blowing method to the DD-screen hung from a hovering drone (C). Adult flies were blown into the airstream from a blower to the screen $(A, C)$, and the number of insects captured with the screen was counted. In $\mathrm{C}$, the position in which the DD-screen was hung varied between $0.5-2 \mathrm{~m}$ from the drone. Abbreviations: $\mathrm{BL}$, blower; MPI, moving path of an insect blown into the airstream; HAC, horizontal air current from a blower; CWT, cylindrical wind tunnel; EF, electric field; DD-S, DD-screen (cross-sectional view); NCT, negatively charged tube; PCT, positively charged tube; SS, slipstream from rotating propellers.

This insect-capturing assay was also applied to a DD-screen hung from a drone that was hovered at a height of $3 \mathrm{~m}$ (Figure 6C). In this experiment, test flies were blown at $8 \mathrm{~m} / \mathrm{s}$ toward a DD-screen that was oppositely charged with a voltage of $6.5 \mathrm{kV}$. In this assay, the DD-screen was hung at positions of $0.5,1,1.5$, and $2 \mathrm{~m}$ below the drone.

\subsection{Trial Flight under Field Conditions}

The newly constructed DD-screen was hung at a position $1.8 \mathrm{~m}$ below the drone (Figure 4B) and then used for trial flights under field conditions. In the first experiment, an anemometer was integrated into the DD-screen hung from the drone, and the DDscreen-carrying drone (1D-drone) was flown at $5 \mathrm{~km} / \mathrm{h}$ under conditions of an upwind or downwind airflow of $5 \mathrm{~m} / \mathrm{s}$. This was the maximum wind speed under which the drone was flown for insect monitoring and was used to measure the maximum speed of the L-airflow that the DD-screen received during a flight.

In the second experiment, we conducted several flights (flight speed, $5 \mathrm{~km} / \mathrm{h}$; endurance, $20 \mathrm{~min}$; wind speed, $<5 \mathrm{~m} / \mathrm{s}$ ) to confirm the stable flight of the 1D-drone under different wind conditions and to examine the kind and number of airborne insects trapped in each flight. The 1D-drone was flown over trellised grape vines (approximate height of trellis, $2 \mathrm{~m}$ ) in an orchard and over blueberry trees (plant height, 1-1.2 m) and basil plants (plant height, 30-50 cm) grown in a field. The drone was operated so as to fly without contacting the DD-screen against the plants (non-touching flight) or to fly while gently touching the upper parts of the plants with the DD-screen (plant-touching flight). The captured insects were classified into four groups: large flies similar to the size of adult houseflies (L-flies); medium flies similar to the size of vinegar flies (M-flies); small flies similar to the size of tomato leaf miners (S-flies) (Figure 5); others, which included whiteflies, winged aphids, thrips, mosquitoes, and stink bugs. The trapped insects were not examined for any further information. 


\subsection{Statistical Analysis}

All experiments were repeated five times, and data are presented as the mean and standard deviation. Analyses were performed to identify significant differences among conditions, as shown in the figure and table legends. For this purpose, Tukey's test was conducted using the EZR software version 1.54 (Jichi Medical University, Saitama, Japan).

\section{Results and Discussion}

A drone is a UAV that is either operated by a human from a remote location or works autonomously as per a set mode. Filho et al. [32] reviewed the effectiveness of advanced imaging technologies based on unmanned aerial robots (small drones) in precision pest management. Hardy et al. [33] conducted a drone-based survey of mosquito aquatic habitats from high-resolution georeferenced images for effective larval source management. Venegas et al. [23] proposed a new approach of remotely sensed image processing combined with UAV techniques for detecting and predicting the expansion of a Phylloxera infestation. Recently, Roosejen et al. [28] reported a unique approach of insect monitoring based on camera images of fruit flies caught on adhesive insect traps, which were taken by a remotecontrolled flying UAV. Although these are all UAV-based remote-photographic techniques, they are not a direct approach for collecting and analysing individual insects or their populations. In an epidemiological survey of pest expansion, trapping-based surveillance of potential insect pests is essential to trace their movement throughout the areas they occupy during their life cycle. In the case of fruit flies, there are many places that need to be surveyed through rapid and effective trapping of the target insects. The DD-screen-carrying drone was designed to reduce the time, cost, and labour of insect-trapping operations.

\subsection{The Propeller Slipstream Disturbs the Longitudinal Airflow toward the DD-Screen Carried by the Drone}

A quadcopter is able to perform stable flight (i.e., ascending and descending, hovering, moving forward and backward, and turning left and right) by the aerodynamic lift and thrust generated by the controlled continuous rotation of four propellers. Two propellers rotate clockwise and the other two rotate counter-clockwise, creating a net torque about the yaw axis zero that maintains the balanced attitude of the aircraft [34]. This means that the four propellers continuously generate a strong downward airstream (slipstream) during the entire flight operation [26]. There was a concern in this study that this downward airstream would intercept the L-airflow (Figure 2B) that drives airborne insects to the DD-screen.

Figure $\mathrm{S} 1$ shows the flow speed of the propeller slipstream that was measured with an anemometer positioned in the front of and at different distances from the drone. In this experiment, the drone was controlled to hover at $3 \mathrm{~m}$ above the ground, at which height there was no influence from the upward airflow that was generated by the rebound of the slipstream from the ground. During operation, the four propellers synchronously increased (decreased) their rotation rate for ascending (descending) and maintained a fixed rotation rate to hover at the target height [34]. Faster propeller rotation produced a stronger slipstream, and the slipstream produced by the four propellers acted as a strong air-barrier in the space beneath all four sides of the drone. It was found that the slipstream-barrier extended to approximately $2 \mathrm{~m}$ from the drone, although the speed of the slipstream decreased with increased distance from the drone. This air-barrier was retained during the operation of the drone. Accordingly, it was likely that the propeller slipstream would impede the L-airflow more strongly in the area close to the drone's body.

To assess this possibility, an anemometer was set at fixed positions beneath the central point of the drone, which was the point from which the DD-screen was hung and received the L-airflow produced by the forward-moving drone. In this experiment, the L-airflow was produced by a blower, and a horizontal airflow was blown to the anemometer at the different positions (Table 1). We calculated the volumetric flow rate from the airflow speed to estimate the inrush of the L-airflow into the area beneath the drone. The air-barrier of the slipstream extended approximately $2 \mathrm{~m}$ downward (Figure S1). This experiment 
indicated that the L-airflow was largely prevented from entering this area (Table 1). At $0.5 \mathrm{~m}$ from the drone, the influence of the slipstream was high enough to impede the entry of more than $90 \%$ of the air. The influence of the slipstream decreased as the distance from the drone increased. Eventually, at $2 \mathrm{~m}$, more than $80 \%$ of the L-airflow was able to enter this area. These results supported our supposition that the upper area beneath the drone was surrounded by the strong slipstream of the four propellers. It was apparent that a DD-screen hung within this area would receive little or no airflow. On the other hand, at around $2 \mathrm{~m}$, the influence of the slipstream was so weak that it could be neglected. Table 1 indicates that as the speed of the L-airflow increased, larger amounts of air reached the area beneath the drone.

Table 1. Changes in speed and volumetric flow rate of a horizontal airflow (L-airflow) blown toward an anemometer set at definite positions beneath a hovering drone.

\begin{tabular}{|c|c|c|c|c|}
\hline $\begin{array}{c}\text { Speed of L-Airflow } \\
\text { Produced by a Blower } \\
(\mathrm{m} / \mathrm{s})\end{array}$ & Position (m) of AM ${ }^{I}$ & $\begin{array}{c}\text { Speed (m/s) of } \\
\text { L-Airflow at the Site } \\
\text { of AM }\end{array}$ & $\begin{array}{c}\text { Volumetric Flow Rate } \\
\left(\mathrm{m}^{3} / \mathrm{min}\right) \text { at the Site of } \\
\text { AM }\end{array}$ & $\begin{array}{c}\text { Percentage of } \\
\text { Volumetric Flow Rate } \\
\text { in AM }\end{array}$ \\
\hline \multirow{4}{*}{2} & 0.5 & $0.14 \pm 0.05$ & $0.13 \pm 0.05$ & $4.7 \pm 1.8 \mathrm{a}$ \\
\hline & 1 & $0.18 \pm 0.04$ & $0.16 \pm 0.04$ & $6.0 \pm 1.5 \mathrm{a}$ \\
\hline & 1.5 & $0.88 \pm 0.19$ & $0.79 \pm 0.17$ & $29.3 \pm 6.4 c$ \\
\hline & 2 & $2.46 \pm 0.35$ & $2.21 \pm 0.32$ & $82.0 \pm 11.7 \mathrm{e}$ \\
\hline \multirow{4}{*}{5} & 0.5 & $0.14 \pm 0.11$ & $0.13 \pm 0.10$ & $2.8 \pm 2.3 \mathrm{a}$ \\
\hline & 1 & $0.66 \pm 0.44$ & $0.59 \pm 0.40$ & $12.4 \pm 3.8 \mathrm{~b}$ \\
\hline & 1.5 & $2.38 \pm 0.64$ & $2.14 \pm 0.57$ & $47.6 \pm 12.8 \mathrm{~d}$ \\
\hline & 2 & $4.26 \pm 0.30$ & $3.83 \pm 0.27$ & $85.2 \pm 6.1 \mathrm{e}$ \\
\hline \multirow{4}{*}{8} & 0.5 & $0.48 \pm 0.26$ & $0.43 \pm 0.23$ & $6.0 \pm 3.2 \mathrm{a}$ \\
\hline & 1 & $1.14 \pm 0.34$ & $1.03 \pm 0.30$ & $14.3 \pm 4.2 \mathrm{~b}$ \\
\hline & 1.5 & $5.36 \pm 1.04$ & $4.82 \pm 0.93$ & $67.0 \pm 12.9 \mathrm{~d}$ \\
\hline & 2 & $7.86 \pm 0.39$ & $7.07 \pm 0.35$ & $98.3 \pm 2.1 \mathrm{f}$ \\
\hline
\end{tabular}

${ }^{\mathrm{I}}$ While the drone hovered at a height of $3 \mathrm{~m}$, the L-airflow was blown toward an anemometer (AM) set at different positions beneath the central point of the drone body. II Percentage of the L-airflow that reached the AM, relative to the volumetric flow rate in the absence of a slipstream. The means and standard deviations were calculated from five repetitions of the experiments. The letters ( $a-f)$ on the means in a vertical column indicate significant differences $(p<0.05)$ according to a Tukey's test.

\subsection{The Drone Could Carry the Newly Devised DD-Screen in a Remote-Controlled Flight}

A voltage generator was an essential part of the DD-screen. It was used to boost the initial voltage $(4.5 \mathrm{~V})$ to the designated voltage $(2-7 \mathrm{kV})$ using a transformer (coil) and a Cockcroft circuit [35] integrated into an electric circuit in the voltage generator. Figure S2 shows the electric circuit configuration for voltage charging to the DD-screen. In a conventional DD-screen, a negative voltage generator draws negative electricity, free electrons, from the ground (an infinite source or sink of electrons-in this case, a source) and supplies electrons to the conductor (negative electrification). In contrast, a positive voltage generator pushes the free electrons of the conductor to the ground (an infinite sink of electrons) to positively charge the conductor (positive electrification) (Figure S2A) [36]. In the non-grounded circuit used in this study, the free electrons of the conductor were supplied directly to another conductor using the voltage produced by two voltage generators that were unified in one box (Figure S2B) [36]. Therefore, with this circuit, the DD-screen has no need for an earth-ground line. The electric field screen placement is freely selectable, allowing a portable electric field screen [37]. Eventually, the DD-screen could be applied to a drone to trap airborne insects during flight. In this study, we set an objective of attaining an endurance of more than $21 \mathrm{~min}$, which was $20 \%$ less than the duration of a non-payload flight of the drone.

In the preliminary experiment, we attached a conventional DD-screen to the drone to determine whether the drone could carry it. It was found that the weight $(1.2 \mathrm{~kg})$ of the DD-screen exceeded the permissible limit, and therefore, the drone could not lift the screen during its ascent. It, therefore, became essential to fabricate a lightweight DD-screen to 
realise drone-based monitoring of airborne insects. In a conventional DD-screen, multiple iron wires are passed through insulating tubes for electrification, and these metal materials account for approximately $70 \%$ of the total weight of the DD-screen. A conductive chemical paste was applied to the inner surface of a PVC tube as a metal alternative. When dry, this successfully reduced the weight to $40 \%$ of the original weight. In addition, two voltage generators were miniaturised and unified. The voltage generator used in a conventional DD-screen is operated by a 12-volt lithium battery [12], which is three-times heavier than the three size AA batteries used in the screen developed in this study. Eventually, the weight of the new DD-screen was sufficiently reduced ( $475 \mathrm{~g}$ ) that the drone could carry it during a remote-controlled flight. Figure S3 shows the effect of flight speed on the endurance of the drone carrying the DD-screen with four different screen-hanging styles. There was no significant difference in flight endurance among drone flights carrying the DD-screen in different positions over the range of flight speeds $(5-20 \mathrm{~km} / \mathrm{h})$ tested. Figure $\mathrm{S} 4$ is a photographic demonstration of a successful flight of the drone carrying the DD-screen in different positions. It was possible to achieve the objective flight time (more than $21 \mathrm{~min}$ ) for the drone carrying the DD-screen.

A drone is fundamentally vulnerable to wind and can crash in strong winds [34]. Drone manufacturers recommend that a drone should be flown only on calm days or in a light breeze $(5-7 \mathrm{~m} / \mathrm{s})$. In addition, there is also a risk that the L-airflow produced by a moving drone at high speed can cause it to lose its normal attitude during flight [34]. Considering these potential negative factors, we finally established the most suitable conditions for an insect-monitoring flight: payload, single DD-screen; position of the DD-screen attachment, $1.8 \mathrm{~m}$ from the drone body; flight speed, $5 \mathrm{~km} / \mathrm{h}$; wind conditions for flight performance, less than $5 \mathrm{~m} / \mathrm{s}$. In addition, taking precautions against a downwash (rebound of the slipstream against the ground surface) was of special importance to avoid crashing especially when the drone was near the ground. For this purpose, slow ascending/descending movements of the drone were essential to maintain the normal altitude of the drone against the downwash.

\subsection{The DD-Screen Can Capture Airborne Insects in Windy Conditions}

Under windless conditions, the DD-screen attached to a drone receives the L-airflow at the same speed as the flight speed of the drone (i.e., approximately $1.38 \mathrm{~m} / \mathrm{s}$ at $5 \mathrm{~km} / \mathrm{h}$ ) [38]. However, in outdoor flight operation, the L-airflow is affected by natural wind, and therefore, it is necessary to calculate the resultant wind speed by adding or subtracting the natural wind speed to or from the wind-free airflow speed for the head- and tail-wind, respectively [38]. In our study, the drone was intended to be flown in a maximum headand tail-wind of $5 \mathrm{~m} / \mathrm{s}$, and therefore, the DD-screen would be exposed to an L-airflow of $6.3 \mathrm{~m} / \mathrm{s}$ and a reverse airflow of $3.7 \mathrm{~m} / \mathrm{s}$ under head- and tail-wind conditions, respectively. This simulation implies that we should give the DD-screen the ability to capture insects that are carried by winds of different speeds $(2-8 \mathrm{~m} / \mathrm{s})$. The capture site of a DD-screen is an electric field formed between opposite poles (in this study, negatively and positively charged insulator tubes) (Figures $3 \mathrm{~A}$ and $6 \mathrm{~B}$ ), and insects that enter the electric field are attracted to the closest pole [12,14]. In this experiment, we determined the ability of the newly constructed DD-screen to capture insects that were blown toward the screen at different wind speeds.

Table 2 lists the percentage of test insects (adult houseflies, vinegar flies, and tomato leaf miners) captured by the DD-screen at various voltages in the range of $2-7 \mathrm{kV}$, and at various wind speeds in the range of $2-8 \mathrm{~m} / \mathrm{s}$. Higher voltages had to be applied to the tubes to capture insects with larger body sizes, and higher voltages were required at higher wind speeds. The force of the charged tubes became stronger as the voltage applied to the tubes increased. For voltages in excess of $6.5 \mathrm{kV}$, the tubes captured all of the insects at all wind speeds investigated. The electrostatic forces were sufficient to capture the insects at wind speeds of up to $8 \mathrm{~m} / \mathrm{s}$. 
Table 2. Capture of test insects blown at different wind speeds towards the DD-screen charged with different voltages.

\begin{tabular}{|c|c|c|c|c|c|c|c|c|c|}
\hline \multirow{2}{*}{$\begin{array}{c}\text { Wind } \\
\text { Speed }(\mathrm{m} / \mathrm{s})\end{array}$} & \multirow{2}{*}{ Test Insect } & \multicolumn{8}{|c|}{ Negative and Positive Voltages (kV) Applied to DD-Screen } \\
\hline & & 2 & 3 & 4 & 5 & 5.5 & 6 & 6.5 & 7 \\
\hline \multirow{3}{*}{2} & TLM & 0 & $90.2 \pm 6.7 \mathrm{a}$ & $100 \mathrm{a}$ & $100 \mathrm{a}$ & $100 \mathrm{a}$ & $100 \mathrm{a}$ & 100 & 100 \\
\hline & VF & 0 & $51.9 \pm 3.3 b$ & $93.6 \pm 8.5 b$ & $100 \mathrm{a}$ & $100 \mathrm{a}$ & $100 \mathrm{a}$ & 100 & 100 \\
\hline & HF & 0 & $12.4 \pm 3.4 \mathrm{c}$ & $31.9 \pm 2.3 c$ & $91.2 \pm 4.6 \mathrm{~b}$ & $100 \mathrm{a}$ & $100 \mathrm{a}$ & 100 & 100 \\
\hline \multirow{3}{*}{5} & TLM & 0 & $44.7 \pm 2.3 b$ & $95.5 \pm 5.7 b$ & $100 \mathrm{a}$ & $100 \mathrm{a}$ & $100 \mathrm{a}$ & 100 & 100 \\
\hline & $\mathrm{VF}$ & 0 & $8.2 \pm 0.7 \mathrm{~d}$ & $22.2 \pm 8.7 \mathrm{~d}$ & $93.8 \pm 7.8 b$ & $100 \mathrm{a}$ & $100 \mathrm{a}$ & 100 & 100 \\
\hline & HF & 0 & $0 \mathrm{e}$ & $6.2 \pm 0.5 \mathrm{e}$ & $31.9 \pm 6.3 c$ & $89.2 \pm 6.2 b$ & $100 \mathrm{a}$ & 100 & 100 \\
\hline \multirow{3}{*}{8} & TLM & 0 & $10.2 \pm 0.7 \mathrm{~d}$ & $41.7 \pm 7.5 c$ & $96.4 \pm 8.6 b$ & $100 \mathrm{a}$ & $100 \mathrm{a}$ & 100 & 100 \\
\hline & VF & 0 & $0 \mathrm{e}$ & $7.7 \pm 0.5 \mathrm{e}$ & $42.7 \pm 5.6 c$ & $96.4 \pm 8.5 b$ & $100 \mathrm{a}$ & 100 & 100 \\
\hline & $\mathrm{HF}$ & 0 & $0 \mathrm{e}$ & $0 \mathrm{f}$ & $9.2 \pm 0.7 \mathrm{~d}$ & $43.8 \pm 6.3 c$ & $91.2 \pm 2.7 b$ & 100 & 100 \\
\hline
\end{tabular}

Adult specimens of tomato leaf miner (TLM), vinegar fly (VF), and housefly (HF) were used as test insects. Twenty insects were used for tests with each voltage, each species, and each wind speed, and the means and standard deviations were calculated from five repetitions. The letters $(\mathrm{a}-\mathrm{f})$ on the means in each vertical column indicate significant differences $(p<0.05)$ according to a Tukey's test.

Video S1 demonstrates the successful capture of three test flies blown toward the DD-screen at $8 \mathrm{~m} / \mathrm{s}$. These results demonstrate that the DD-screen at $6.5 \mathrm{kV}$ could trap all of the major pest insects investigated under real wind conditions during the flight. However, at lower voltages, the electrostatic forces were not sufficient to capture the insects. The flies moved their legs, twisted their bodies, and then flew away from the charged tube, or they were blown away from the tube by the blower. Based on these observations, in the subsequent field flight, a voltage of $6.5 \mathrm{kV}$ was applied to ensure the successful capture of the insects at wind speeds of up to $8 \mathrm{~m} / \mathrm{s}$. This ensured that the insects were captured at the maximum L-airflow speed that the DD-screen experienced.

The study demonstrated that the newly constructed DD-screen was able to capture airborne insects carried by a wind of $8 \mathrm{~m} / \mathrm{s}$. This wind speed was equivalent to the Lairflow produced by a drone flying at approximately $29 \mathrm{~km} / \mathrm{h}$ (windless conditions). To ensure the capture of the insects, it was essential that the airborne insects were carried to the DD-screen by the L-airflow. Therefore, the results presented in Table 1, indicating that the slipstream could impede the inrush of the L-airflow, were important to consider when determining the position of the DD-screen that ensured a sufficient L-airflow for the DD-screen during the flight of the drone. To solve this problem, we conducted an additional capturing assay using the method shown in Figure $6 \mathrm{C}$, and the results are shown in Table 3. When the DD-screen was positioned at 0.5 and $1 \mathrm{~m}$, all of the test insects blown toward the DD-screen at 2 and $5 \mathrm{~m} / \mathrm{s}$ were blown downward at the position of the slipstream, and therefore, no insects reached the DD-screen. This occurred for all insect species. However, when the insects were blown at $8 \mathrm{~m} / \mathrm{s}$, a small number of flies did reach the DD-screen positioned at $1 \mathrm{~m}$ below the drone, although no fly reached the DD-screen at $0.5 \mathrm{~m}$. With the DD-screen at $1.5 \mathrm{~m}$, the number of insects reaching the DD-screen increased in response to the increase in the blow speed. More than $90 \%$ of the insects reached the DD-screen at $2 \mathrm{~m}$, regardless of the difference in blow speed. Judging from these results, we hung the DD-screen at $1.8 \mathrm{~m}$ from the drone, enabling the screen surface to cover the range of $1.85-2 \mathrm{~m}$ beneath the drone.

\subsection{A Drone-Carried DD-Screen Is an Effective Tool to Capture Airborne Insects during a Remote-Controlled Flight}

In the final experiment, we conducted several trial flights of the 1D-drone under different wind conditions. First, we measured the L-airflow that passed through the DDscreen during the flight with an upwind of $5 \mathrm{~m} / \mathrm{s}$ to compare the flow speed values obtained by the actual measurement with the values obtained from the calculation mentioned above. In all tests, during a flight of $21 \mathrm{~min}$, the wind speed $(5 \mathrm{~m} / \mathrm{s})$ at the start of the flight changed hour by hour in the range of $1.2-7.3 \mathrm{~m} / \mathrm{s}$ and $0.3-2.4 \mathrm{~m} / \mathrm{s}$ under the head- and tail-wind conditions, respectively. These wind speeds were apparently within the normal 
tolerance of the DD-screen charged with $6.5 \mathrm{kV}$. The DD-screen was actually able to capture airborne insects carried by a wind of $8 \mathrm{~m} / \mathrm{s}$ (Table 2).

Table 3. Capture of test insects blown toward the DD-screen hung from the drone at different positions.

\begin{tabular}{ccccc}
\hline \multirow{2}{*}{$\begin{array}{c}\text { Blow Speed } \\
(\mathbf{m} / \mathbf{s})\end{array}$} & $\begin{array}{c}\text { Position }(\mathbf{m}) \text { of } \\
\text { DD-Screen }\end{array}$ & \multicolumn{3}{c}{ Percentage of Test Insects Captured with DD-Screen Oppositely Charged with 6.5 kV } \\
\cline { 3 - 5 } & 0.5 & Tomato Leaf Miners & Vinegar Flies & Houseflies \\
\hline & 1 & $0 \mathrm{a}$ & $0 \mathrm{a}$ & $0 \mathrm{a}$ \\
& 1.5 & $0 \mathrm{a}$ & $0 \mathrm{a}$ & $0.9 \pm 2.5 \mathrm{~b}$ \\
& 2 & $5.2 \pm 3.6 \mathrm{~b}$ & $1.9 \pm 7.3 \mathrm{~b}$ & $09.4 \pm 4.9 \mathrm{~d}$ \\
\hline & 0.5 & $93.6 \pm 3.6 \mathrm{~d}$ & $02.9 \pm 4.5 \mathrm{~d}$ & $0 \mathrm{a}$ \\
& 1 & $0 \mathrm{a}$ & $0 \mathrm{a}$ & $50.7 \pm 6.2 \mathrm{c}$ \\
& 1.5 & $0 \mathrm{a}$ & $65.9 \pm 7.3 \mathrm{c}$ & $91.2 \pm 4.8 \mathrm{~d}$ \\
\hline & 0.5 & $94.7 \pm 3.7 \mathrm{c}$ & $93.6 \pm 3.8 \mathrm{~d}$ & $0 \mathrm{a}$ \\
& 1 & $0 \mathrm{a}$ & $0 \mathrm{a}$ & $5.6 \pm 3.6 \mathrm{~b}$ \\
& 1.5 & $12.1 \pm 7.9 \mathrm{~b} \mathrm{~d}$ & $10.9 \pm 7.3 \mathrm{~b}$ & $86.5 \pm 3.3 \mathrm{~d}$ \\
& 2 & $92.1 \pm 2.4 \mathrm{~d}$ & $90.9 \pm 1.3 \mathrm{~d}$ & $93.9 \pm 2.7 \mathrm{~d}$ \\
\hline
\end{tabular}

A DD-screen was hung at different positions from a drone that was hovered at a height of $2 \mathrm{~m}$, and the horizontal L-airflow was blown toward each DD-screen. Test insects were blown into the airstream driven to the DD-screen. Twenty insects were used for tests with each species and each blow speed, and the means and standard deviations were calculated from five repetitions. The letters (a-e) on the means in each vertical column indicate significant differences $(p<0.05)$ according to a Tukey's test.

Successful capture of airborne insects with the drone-carried DD-screen depends on the presence of target insects in the path of the DD-screen. Mosquito columns (midge swarms), which occur as a consequence of the collective behaviour of male mosquitoes to attract females for mating [39], were a good target to confirm the ability of the DD-screen to capture numerous insects in the air [1]. However, at a height of more than $3 \mathrm{~m}$ (height of the DD-screen), airborne insects were seldom encountered during the flight. To solve this problem, the test area of the flight was changed to a location in the close vicinity of horticultural plants grown in crop fields and orchards. Video S2 shows the flight of the 1D-drone, with insects captured using the two methods (non-plant-touching and planttouching). Table $4 \mathrm{~A}$ provides the insect collection results of several flights of the 1D-drone. In this experiment, we used the two capture methods and collected insects in the vicinity of trellised and non-trellised vines in a vineyard and blueberry plants grown in a field, both of which produced ripe fruits, as well as topped basil plants growing from axillary buds in a field. These three plants were grown in an area of $20 \times 20 \mathrm{~m}^{2}$. In the non-plant-touching method, a few M-flies (similar to fruit flies) were caught around the grape vines and blueberry plants. These flies were deemed to have been captured after leaving the ripe fruits of the target trees. Around the basil plants, S-flies were occasionally trapped. These flies were considered to be leaf miners that visited basil plants for oviposition, because typical mines were observed in many leaves of the basil plants.

It has been observed that pest insects frequently ascend from their nesting positions when the plants are gently tapped. We, therefore, flew the 1D-drone while touching the target plants with the DD-screen carried by the drone (Table 4B). This encouraged L-flies to fly up from the grape vines, blueberry plants, and basil plants; they could then be captured with the DD-screen in large numbers during the flight. In addition, large numbers of whiteflies were simultaneously captured from the basil plants when this method was applied. These pests were confirmed to be nesting on the basil plants. In both approaches (non-plant-touching and plant-touching methods), we trapped similar numbers of L-flies from basil plants (Table 4A,B). Of note, the L-flies were frequently trapped when the 1Ddrone was flown over non-planted ridges in a field at a height of $30 \mathrm{~cm}$ (data not shown). The ridge soil was fertilised with cattle manure. Cattle manure has been reported to contain the larvae and pupae of houseflies [40]. We frequently detected houseflies emerging from underground pupae. The drone flight, therefore, collected these houseflies flying up from 
the ground. In other flights, we trapped various species of insects, such as houseflies, fruit flies, leaf miners, white flies, winged aphids, thrips, biting mosquitoes, midges, and stink bugs [1]. The results obtained from the trial flights demonstrated that the drone-carried DD-screen was able to successfully trap a wide range of airborne insects using two different methods and provides an experimental basis for the trap-based monitoring of airborne insects. Incidentally, the insects that were trapped with the DD-screen were alive even after they were continuously captured for $1 \mathrm{hr}$. The insects remained motionless for 1-2 min after the voltage supply was cut and then walked around or flew away. This situation was useful to assort the trapped insects for specified purposes or to safely release beneficial insects such as pollinators and natural enemies of insect pests.

Table 4. Details of the insects captured with the drone-carried DD-screen during insect-collecting flights I.

\begin{tabular}{|c|c|c|c|c|c|c|}
\hline \multirow{3}{*}{ Experiment ${ }^{\mathrm{II}}$} & \multirow{3}{*}{ Site of Flight } & \multirow{3}{*}{ Total Number of Captured Insects } & \multicolumn{4}{|c|}{ Number of Insects Captured III } \\
\hline & & & \multicolumn{3}{|c|}{ Flies } & \multirow{2}{*}{ Others } \\
\hline & & & $\mathrm{L}$ & $\mathbf{M}$ & $\mathrm{S}$ & \\
\hline \multirow{3}{*}{ A (NFT) } & Trellised vines in orchard & 2 & 0 & 2 & 0 & 0 \\
\hline & Blueberries in field & 4 & 0 & 4 & 0 & 0 \\
\hline & Basils in field & 9 & 6 & 0 & 2 & $1(\mathrm{WF})$ \\
\hline \multirow{3}{*}{$\mathrm{B}(\mathrm{PF})$} & $\begin{array}{c}\text { Non-trellised vines in } \\
\text { orchard }\end{array}$ & 24 & 0 & 24 & 0 & 0 \\
\hline & Blueberries in field & 45 & 0 & 45 & 0 & 0 \\
\hline & Basils in field & 49 & 7 & 0 & 24 & $17(\mathrm{WF})+1(\mathrm{WA})$ \\
\hline
\end{tabular}

I The drone carrying single DD-screen was flown during days with a wind speed of $1-5 \mathrm{~m} / \mathrm{s}$, with a $5 \mathrm{~km} / \mathrm{h}$ flight speed and $25 \mathrm{~min}$ endurance time. II NFT, flight with non-contact of DD-screen with plants; PF, flight while touching plants with DD-screen. III The captured insects were classified into two groups (flies and others), and flies were subdivided into three groups: large flies (L-flies), similar to houseflies; middle flies (M-flies), similar to vinegar flies; small flies (S-flies), similar to tomato leaf miners. Other captured insects included whiteflies (WF) and winged aphids (WA).

\section{Conclusions}

A new approach for direct monitoring of airborne insects was developed based on electrostatic engineering techniques combined with UAV technology. In this electrostatic approach, an electric field screen was used to create an attractive force in a static electric field and capture insects that entered the electric field. The static electric field was formed in the space between oppositely charged parallel insulator tubes with a sufficient voltage. The aim was to construct a lightweight electric field screen to enable a light payload on a drone (quadcopter) and, therefore, ensure sufficient flight endurance for the capture of insects. The application of a conductive coating to the inner surface of the insulator tube and unification of two negative and positive voltage generators were crucial aspects of the newly developed screen. Analyses of the strong downward slipstream created by four revolving propellers and longitudinal airflow generated by a moving drone were essential to determine the most suitable location of the electric field screen attached to the drone. This enabled an insect-carrying air current to pass through the screen during drone flight, and wind-carried insects could be eliminated with the electric field screen when the wind passed through the screen. The electric field screen constructed in the study was functional enough to capture model insects (three flies with different body sizes) carried by a wind of $8 \mathrm{~m} / \mathrm{s}$ Thus, the construction and optimisation of the drone-carried electric field screen made it possible to collect airborne insects during drone flight and provided an experimental basis for a UAV-based insect trapping system.

Supplementary Materials: The followings are available online at https:/ / www.mdpi.com/2077-047 2/11/2/176/s1, Figure S1: Changes in the flow speed of the slipstream produced by the propellers of a quadcopter (drone) hovering at a height of $2 \mathrm{~m}$, Figure S2: Schematic representation of grounded (A) and non-grounded (B) circuits integrated into the DD-screen, Figure S3: Changes in flight endurance (min) of a quadcopter (drone) carrying the DD-screen developed in the study at different flight 
speeds, Figure S4: Successful flight of a drone carrying a DD-screen at positions of 0.5 (A), 1 (B), 1.5 (C), and $2 \mathrm{~m}$ (D) beneath the drone body, Video S1: Capture of test flies with the charged insulator tubes of the DD-screen, Video S2: Remote-controlled flight of the 1D-drone to collect insects using plant-touching (A) and non-plant-touching (B) methods.

Author Contributions: Conceptualization, K.K. and H.T.; methodology, Y.M., Y.T., K.O., and K.K.; software, Y.M.; validation, K.K. and T.N.; formal analysis, T.N.; investigation, Y.M. and K.K.; resources, Y.M.; data curation, K.K. and T.N.; writing—original draft preparation, H.T. and K.K.; writingreview and editing, T.N. and H.T.; visualization, Y.M.; supervision, T.N.; project administration, Y.M.; funding acquisition, Y.M. All authors have read and agreed to the published version of the manuscript.

Funding: This research was funded by JSPS KAKENHI, Grant Number 19K06299.

Conflicts of Interest: The authors declare no conflict of interest.

\section{References}

1. Kusakari, S.; Okada, K.; Shibao, M.; Toyoda, H. High voltage electric fields have potential to create new physical pest control systems. Insects 2020, 11, 447. [CrossRef]

2. Matsuda, Y.; Ikeda, H.; Moriura, N.; Tanaka, N.; Shimizu, K.; Oichi, W.; Nonomura, T.; Kakutani, K.; Kusakari, S.; Higashi, K.; et al. A new spore precipitator with polarized dielectric insulators for physical control of tomato powdery mildew. Phytopathology 2006, 96, 967-974. [CrossRef]

3. Shimizu, K.; Matsuda, Y.; Nonomura, T.; Ikeda, H.; Tamura, N.; Kusakari, S.; Kimbara, J.; Toyoda, H. Dual protection of hydroponic tomatoes from rhizosphere pathogens Ralstonia solanacearum and Fusarium oxysporum f. sp. radicis-lycopersici and airborne conidia of Oidium neolycopersici with an ozone-generative electrostatic spore precipitator. Plant Pathol. 2007, 56, 987-997. [CrossRef]

4. Tanaka, N.; Matsuda, Y.; Kato, E.; Kokabe, K.; Furukawa, T.; Nonomura, T.; Honda, K.; Kusakari, S.; Imura, T.; Kimbara, J.; et al. An electric dipolar screen with oppositely polarized insulators for excluding whiteflies from greenhouses. Crop Prot. 2008, 27, 215-221. [CrossRef]

5. Matsuda, Y.; Nonomura, T.; Kakutani, K.; Takikawa, Y.; Kimbara, J.; Kasaishi, Y.; Kusakari, S.; Toyoda, H. A newly devised electric field screen for avoidance and capture of cigarette beetles and vinegar flies. Crop Prot. 2011, 30, 155-162. [CrossRef]

6. Takikawa, Y.; Matsuda, Y.; Nonomura, T.; Kakutani, K.; Kusakari, S.; Toyoda, H. An electrostatic-barrier-forming window that captures airborne pollen grains to prevent pollinosis. Int. J. Environ. Res. Public Health 2017, 14, 82. [CrossRef]

7. Matsuda, Y.; Takikawa, Y.; Nonomura, T.; Kakutani, K.; Okada, K.; Shibao, M.; Kusakari, S.; Miyama, K.; Toyoda, H. A simple electrostatic device for eliminating tobacco sidestream to prevent passive smoking. Instruments 2018, 2, 13. [CrossRef]

8. Toyoda, H.; Kusakari, S.; Matsuda, Y.; Kakutani, K.; Xu, L.; Nonomura, T.; Takikawa, Y. Electric field screen structures. In An Illustrated Manual of Electric Field Screens: Their Structures and Functions; Toyoda, H., Ed.; RAEFSS Publishing Department: Nara, Japan, 2019; pp. 9-15.

9. Kakutani, K.; Matsuda, Y.; Nonomura, T.; Kimbara, J.; Kusakari, S.; Toyoda, H. Practical application of an electric field screen to an exclusion of flying insect pests and airborne conidia from greenhouses with a good air penetration. J. Agric. Sci. 2012, 4, 51-60. [CrossRef]

10. Nonomura, T.; Matsuda, Y.; Kakutani, K.; Kimbara, J.; Osamura, K.; Kusakari, S.; Toyoda, H. An electric field strongly deters whiteflies from entering window-open greenhouses in an electrostatic insect exclusion strategy. Eur. J. Plant Pathol. 2012, 134, 661-670. [CrossRef]

11. Matsuda, Y.; Nonomura, T.; Kakutani, K.; Kimbara, J.; Osamura, K.; Kusakari, S.; Toyoda, H. Avoidance of an electric field by insects: Fundamental biological phenomenon for an electrostatic pest-exclusion strategy. J. Phys. Conf. Ser. 2015, 646, 0120031-0120034. [CrossRef]

12. Nonomura, T.; Matsuda, Y.; Kakutani, K.; Takikawa, Y.; Kimbara, J.; Osamura, K.; Kusakari, S.; Toyoda, H. Prevention of whitefly entry from a greenhouse entrance by furnishing an airflow-oriented pre-entrance room guarded with electric field screens. $J$. Agric. Sci. 2014, 6, 172-184. [CrossRef]

13. Takikawa, Y.; Matsuda, Y.; Nonomura, T.; Kakutani, K.; Okada, K.; Shibao, M.; Kusakari, S.; Miyama, K.; Toyoda, H. Exclusion of whiteflies from a plastic hoop greenhouse by a bamboo blind-type electric field screen. J. Agric. Sci. 2020, 12, 50-60.

14. Matsuda, Y.; Kakutani, K.; Nonomura, T.; Kimbara, J.; Kusakari, S.; Osamura, K.; Toyoda, H. An oppositely charged insect exclusion screen with gap-free multiple electric fields. J. Appl. Phys. 2012, 112, 116103. [CrossRef]

15. Ruwaimana, M.; Satyanarayana, B.; Otero, V.; Muslim, A.M.; Syafiq, A.M.; Ibrahim, S.; Raymaekers, D.; Koedam, N.; DahdouhGuebas, F. The advantages of using drones over space-borne imagery in the mapping of mangrove forests. PLoS ONE 2018, 13, e0200288. [CrossRef] [PubMed]

16. Forsmoo, J.; Anderson, K.; Macleod, C.J.A.; Wilkinson, M.E.; DeBell, L.; Brazier, R.E. Structure from motion photogrammetry in ecology: Does the choice of software matter? Eco. Evol. 2019, 9, 12964-12979. [CrossRef] 
17. Brady, J.M.; Stokes, M.D.; Bonnardel, J.; Bertram, T.H. Characterization of a quadrotor unmanned aircraft system for aerosolparticle-concentration measurements. Environ. Sci. Technol. 2016, 50, 1376-1383. [CrossRef]

18. Rohi, G.; Ejofodomi, O.; Ofualagba, G. Autonomous monitoring, analysis, and countering of air pollution using environmental drones. Heliyon 2020, 6, e03252. [CrossRef]

19. Ferrández-Pastor, F.J.; García-Chamizo, J.M.; Nieto-Hidalgo, M.; Mora-Martínez, J. Precision agriculture design method using a distributed computing architecture on internet of things context. Sensors 2018, 18, 1731. [CrossRef]

20. Maes, W.H.; Steppe, K. Perspectives for remote sensing with unmanned aerial vehicles in precision agriculture. Trends Plant Sci. 2019, 24, 152-164. [CrossRef] [PubMed]

21. Klosterman, S.; Richardson, A.D. Observing spring and fall phenology in a deciduous forest with aerial drone imagery. Sensors 2017, 17, 2852. [CrossRef]

22. Poblete, T.; Ortega-Farías, S.; Ryu, D. Automatic coregistration algorithm to remove canopy shaded pixels in UAV-borne thermal images to improve the estimation of crop water stress index of a drip-irrigated cabernet sauvignon vineyard. Sensors $2018,18,397$. [CrossRef]

23. Vanegas, F.; Bratanov, D.; Powell, K.; Weiss, J.; Gonzalez, F. A novel methodology for improving plant pest surveillance in vineyards and crops using UAV-based hyperspectral and spatial data. Sensors 2018, 18, 260. [CrossRef]

24. Uddin, M.A.; Mansour, A.; Jeune, D.L.; Ayaz, M.; Aggoune, E.-H.M. UAV-assisted dynamic clustering of wireless sensor networks for crop health monitoring. Sensors 2018, 18, 555. [CrossRef] [PubMed]

25. Qin, W.; Qiu, B.; Xue, X.; Chen, C.; Xu, Z.; Zhou, Q. Droplet deposition and control effect of insecticides sprayed with an unmanned aerial vehicle against plant hoppers. Crop Prot. 2016, 85, 79-88. [CrossRef]

26. Wu, Y.; Qi, L.; Zhang, H.; Musiu, E.M.; Yang, Z.; Wang, P. Design of UAV downwash airflow field detection system based on strain effect principle. Sensors 2019, 19, 2630. [CrossRef]

27. Cloonan, K.R.; Abraham, J.; Angeli, S.; Syed, Z.; Rodriguez-Saona, C. Advances in the chemical ecology of the spotted wing drosophila (Drosophila suzukii) and its applications. J. Chem. Ecol. 2018, 44, 922-939. [CrossRef] [PubMed]

28. Roosjen, P.P.J.; Kellenberger, B.; Kooistra, L.; Green, D.R.; Fahrentrapp, J. Deep learning for automated detection of Drosophila suzukii: Potential for UAV-based monitoring. Pest Manag. Sci. 2020, 76, 2994-3002. [CrossRef] [PubMed]

29. Harmon, D.S.; Haseeb, M.; Kanga, L.H.B.; Liburd, O.E. Evaluation of monitoring traps and lures for Drosophila suzukii (Diptera: Drosophilidae) in berry plantings in Florida. Insects 2019, 10, 313. [CrossRef]

30. Zheng, Y.; Yang, S.; Liu, X.; Wang, J.; Norton, T.; Chen, J.; Tan, Y. The computational fluid dynamic modeling of downwash flow field for a six-rotor UAV. Front. Agr. Sci. Eng. 2018, 5, 159-167. [CrossRef]

31. Griffith, W.T. Electrostatic phenomena. In The Physics of Everyday Phenomena, a Conceptual Introduction to Physics; Bruflodt, D., Loehr, B.S., Eds.; McGraw-Hill: New York, NY, USA, 2004; pp. 232-252.

32. Filho, F.H.; Heldens, W.B.; Kong, Z.; de Lange, E.S. Drones: Innovative technology for use in precision pest management. J. Econ. Entomol. 2020, 113, 1-25. [CrossRef]

33. Hardy, A.; Makame, M.; Cross, D.; Majambere, S.; Msellem, M. Using low-cost drones to map malaria vector habitats. Parasit. Vectors 2017, 10, 29. [CrossRef] [PubMed]

34. Rupert, B. How to fly quadcopters and rules. In Drones, the Ultimate Guide; Rupert, B., Ed.; CreateSpace Independent Publishing Platform: Scotts Valley, CA, USA, 2017; pp. 18-24.

35. Wegner, H.E. Electrical charging generators. In McGraw-Hill Encyclopedia of Science and Technology, 9th ed.; Geller, E., Moore, K., Well, J., Blumet, D., Felsenfeld, S., Martin, T., Rappaport, A., Wagner, C., Lai, B., Taylor, R., Eds.; The Lakeside Press: New York, NY, USA, 2002; pp. 42-43.

36. Matsuda, Y.; Kakutani, K.; Nonomura, T.; Kimbara, J.; Osamura, K.; Kusakari, S.; Toyoda, H. Safe housing ensured by an electric field screen that excludes insect-net permeating haematophagous mosquitoes carrying human pathogens. J. Phys. Conf. Ser. 2015, 646, 0120021-0120024. [CrossRef]

37. Takikawa, Y.; Matsuda, Y.; Kakutani, K.; Nonomura, T.; Kusakari, S.; Okada, K.; Kimbara, J.; Osamura, K.; Toyoda, H. Electrostatic insect sweeper for eliminating whiteflies colonizing host plants; a complementary pest control device in an electric field screen-guarded greenhouse. Insects 2015, 6, 442-454. [CrossRef] [PubMed]

38. The Physics Classroom Website. The Plane and the Wind. Available online: https://www.physicsclassroom.com/memedia/ vectors/plane.cfm (accessed on 10 November 2020).

39. Van der Vaart, K.; Sinhuber, M.; Reynolds, A.M.; Ouellette, N.T. Mechanical spectroscopy of insect swarms. Sci. Adv. 2019, 10, 5. [CrossRef] [PubMed]

40. Alam, M.J.; Zurek, L. Association of Escherichia coli O157:H7 with houseflies on a cattle farm. Appl. Environ. Microbiol. 2004, 70, 7578-7580. [CrossRef] [PubMed] 